\title{
Acute pacing-induced cardiomyopathy
}

\author{
Talal Alzahrani, Sripooja Satya, Marco Mercader
}

\begin{abstract}
Introduction: Pacing-induced cardiomyopathy (PICM) is defined as a $10 \%$ reduction in the left ventricular ejection fraction over several months to a year after pacemaker implantation, resulting in an ejection fraction of less than $50 \%$. Nonetheless, other causes of cardiomyopathy should be excluded to diagnose patients with this condition. Case Report: A 59-year-old male presented with a third-degree heart block and underwent pacemaker implantation with right ventricular pacing. Subsequently, he manifested with an acute heart failure exacerbation and a reduced ejection fraction within two months of pacemaker implantation. Consequently, he underwent further workup to exclude causes of dilated cardiomyopathy such as acute coronary syndrome, sarcoidosis, hemochromatosis, granulomatosis with polyangiitis, and eosinophilic myocarditis. His left heart catheterization showed worsening in his ejection fraction without any significant coronary stenosis. He had an endomyocardial biopsy, which showed fibroblast proliferation and fibrosis with no evidence of any infiltrative cardiomyopathy. This patient was diagnosed
\end{abstract}

Talal Alzahrani ${ }^{1}$, Sripooja Satya ${ }^{1}$, Marco Mercader $^{2}$ Affiliations: ${ }^{1} \mathrm{MD}$, Department of Internal Medicine, George Washington University, Washington, DC, USA; ${ }^{2} \mathrm{MD}$, FACC, FHRS, Division of Cardiology, George Washington University, Washington, DC, USA.

Corresponding Author: Talal Alzahrani, Department of Internal Medicine, George Washington University, Washington, DC, USA, 401 12th St South, Apt 2110, zip code: 22202, Arlington, VA, US; E-mail: tsa@gwu.edu

Received: 26 July 2016

Accepted: 11 November 2016

Published: 21 December 2016 with pacing-induced cardiomyopathy because other causes of cardiomyopathy were ruled out. Thus, his cardiac pacemaker device was upgraded to Biventricular pacing device with implantable cardioverter defibrillator. Conclusion: This report describes a patient with a severe acute form of pacing-induced cardiomyopathy and discusses the prevention and therapy of this condition.

Keywords: Cardiomyopathy, Pacing, Pacemaker

\section{How to cite this article}

Alzahrani T, Satya S, Mercader M. Acute pacinginduced cardiomyopathy. Case Rep Int 2016;5:48-53.

Article ID: 100031 CRINTTA2016

$$
* * * * * * * * *
$$

doi:10.5348/crint-2016-31-CR-12

\section{INTRODUCTION}

The first pacemaker devices became available in the late 1960 s and have since become the mainstay of therapy for patients with complete heart block [1]. Since that time, pacemaker implantation has risen dramatically because of both the increase in clinical indications for pacemaker use and the aging of the population. This increased use has lowered the mortality rate and improved quality of life among patients with cardiac arrhythmia [2]. Nevertheless, artificial pacing has been associated with detrimental effects on left ventricular function in patients who require right ventricular pacing such as patients with complete heart block [3]. Several animal studies have shown that right ventricular pacing 
reduces tissue perfusion and increases norepinephrine release in myocardial muscles [4]. Furthermore, it results in histological changes that include myofibrillar cellular disarray and dystrophic calcifications [5]. After heart block was induced by ablation and pacemakers were subsequently implanted, such histological changes in animals' hearts develop within 3-4 months. This adverse clinical phenomenon is called pacing induced cardiomyopathy (PICM).

Pacing induced cardiomyopathy is defined as a 10\% reduction in the left ventricular ejection fraction over several months to a year after pacemaker implantation, resulting in an ejection fraction of less than 50\% [6]. Nonetheless, other causes of cardiomyopathy should be excluded to diagnose patients with this condition [6]. Pacing-induced cardiomyopathy has been reported in up to $9 \%$ of patients who have right ventricular pacing and $1 \%$ who have biventricular pacing [7]. Patients with this condition usually present with symptoms of congestive systolic heart failure such as exertional dyspnea, orthopnea, paroxysmal nocturnal dyspnea, and lower extremity edema. A recent study revealed several factors that raise the risk of PICM such as male gender, a low ejection fraction, and a wide QRS [6]. Our report describes a patient with a severe acute form of PICM and discusses the prevention and therapy of this condition.

\section{CASE REPORT}

A 59-year-old Filipino male with a past medical history of hypertension, hyperlipidemia, and asthma, who started having syncopal episodes associated with coughing four years before admission, which was thought to be vasovagal syncope. At the time, no cardiac rhythm abnormalities were appreciated in his electrocardiography, and he was started on new bronchodilators for a presumed asthma. Currently, he started having syncopal episodes again with concern for seizures. He was evaluated by neurology and found to have a normal electroencephalogram (EEG) and no gross intracranial abnormalities on computed tomography (CT) scan of the head without contrast. Therefore, he was referred to cardiology clinic to evaluate his syncope and was found to have new complete heart block with a ventricular escape. He has a remote history of travel to the Philippines, no history of transfusions, recent international travel, no new industrial exposures, medication changes, sick contacts or insect bites. He had significant alcohol use, 4-5 drinks daily for over 40 years. Laboratory examinations revealed persistent eosinophilia for six years. Immunologic workup was negative for JAK2, PDGFRa, PDGFRb, FGFR1, p-ANCA, c-ANCA but showed elevated serum IgE levels. He was also negative for Trypanosoma cruzi and Schistosoma antibodies. Transthoracic echocardiogram (TTE) showed an ejection fraction of $45 \%$, right ventricular systolic pressure (RSVP) $38 \mathrm{mmHg}$, global hypokinesis of the left ventricle and right atrial (RA) pressure of $3 \mathrm{mmHg}$.
Cardiac MRI scan revealed mildly dilated left ventricle, ejection fraction $45 \%$ and diffuse subendocardial enhancement on late gadolinium imaging with some areas of transmural involvement, thought to be due to fibrosis or inflammation (Figure 1). A dual chamber Biotronik DDD pacemaker was placed, and he continued followup with cardiology clinic with his device functioning appropriately.

The patient subsequently presented two months after pacemaker placement with worsening shortness of breath with exertion over a period of few weeks along with palpitations and anxiety. His repeat echocardiogram
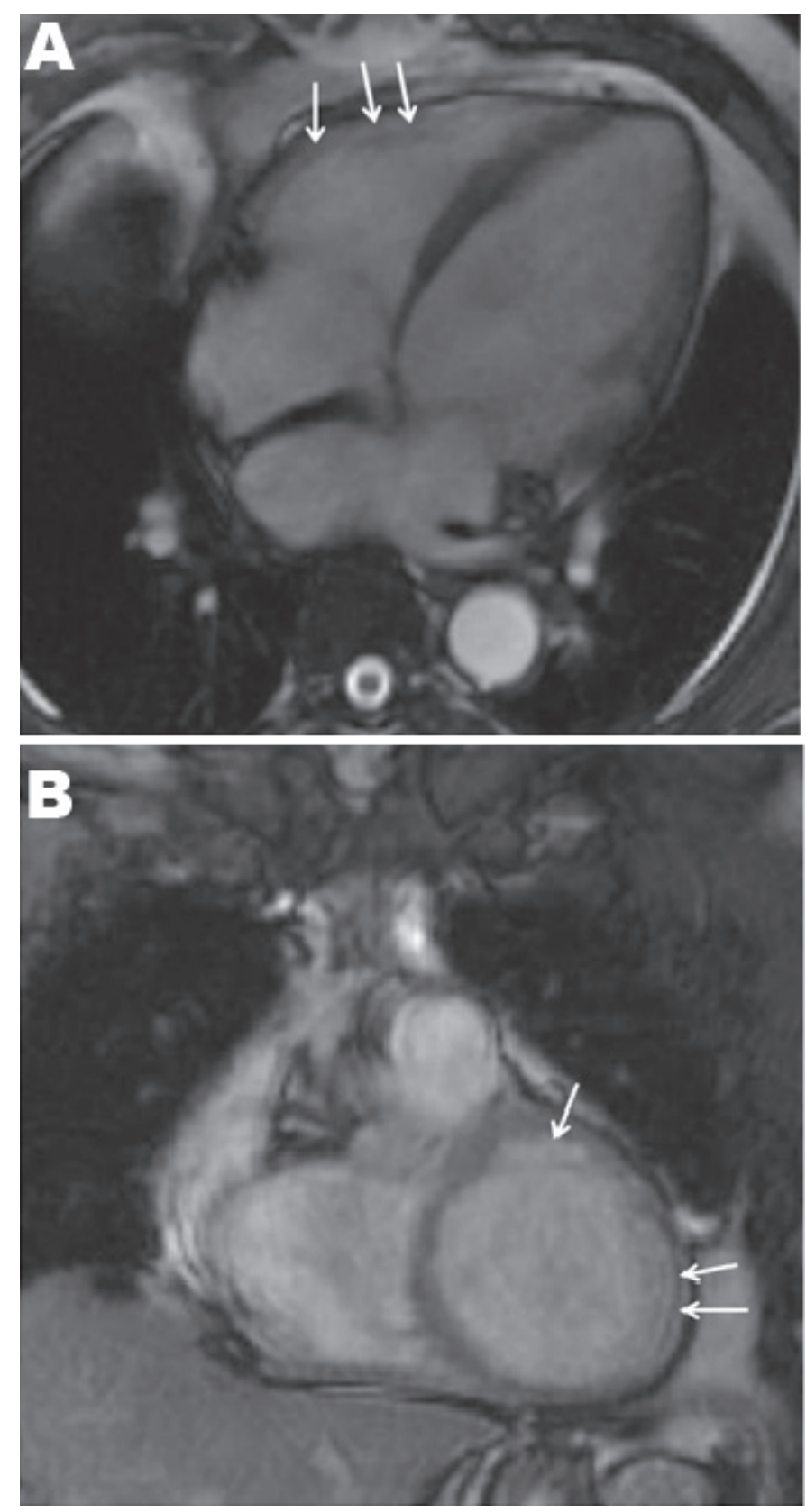

Figure 1: (A, B) Cardiac magnetic resonance imaging scan showing dilated left ventricle, diffuse subendocardial enhancement on late gadolinium imaging with some areas of transmural involvement, likely due to fibrosis or inflammation. 
showed a severely dilated left ventricle and left atrium, moderately dilated right ventricle, severely reduced left ventricular systolic function with an ejection fraction 20-25\%, moderately reduced right ventricular systolic function, septal and apical akinesis with RVSP 30-40 $\mathrm{mmHg}$ and right atrial pressure $15 \mathrm{mmHg}$. Following aggressive diuresis to euvolemia, he was discharged with furosemide, high dose atorvastatin, metoprolol to followup with heart failure clinic for continued management and workup. He then underwent an elective left heart catheterization as an outpatient, which showed ejection fraction of $15 \%$, end diastolic pressure $30 \mathrm{mmHg}$, luminal irregularities in the left anterior descending artery but coronaries otherwise non-obstructive coronary artery disease with right dominant circulation. Right heart catheterization revealed Fick cardiac output of $2.7 \mathrm{~L} /$ min and Fick cardiac index of $1.5 \mathrm{~L} / \mathrm{min} / \mathrm{m}^{2}$, pulmonary capillary wedge pressure (PCWP) $30 \mathrm{mmHg}$, mean pulmonary artery pressure $33 \mathrm{mmHg}$. Endomyocardial biopsy was obtained and revealed fibroblast proliferation and fibrosis but otherwise unremarkable myocardial cells (Figure 2). Congo red staining was negative for
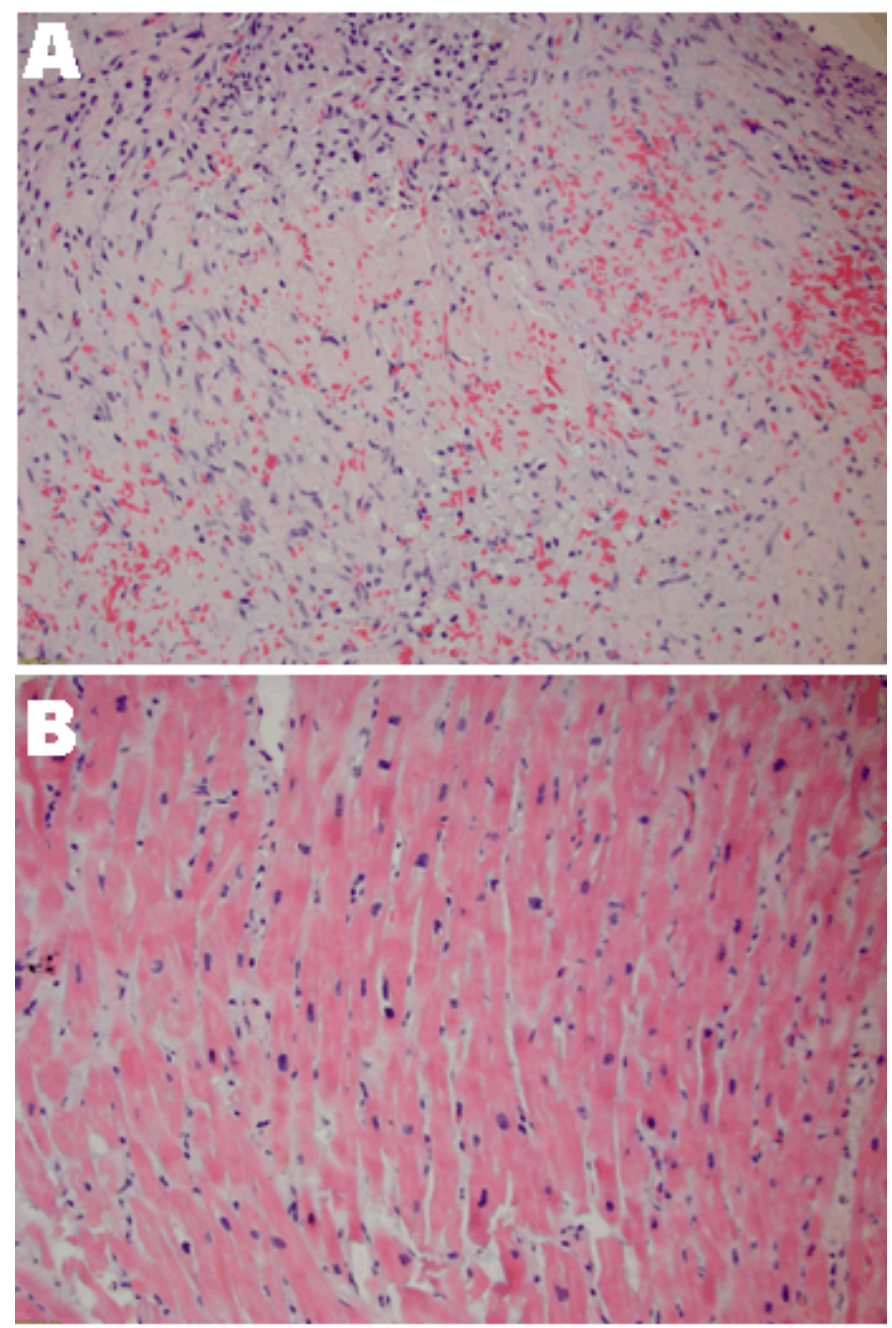

Figure 2: (A, B) Endomyocardial biopsy specimen, fibroblast proliferation, and fibrosis but otherwise unremarkable myocardial cells. amyloid disease. This patient was diagnosed with PICM because other causes of cardiomyopathy were ruled out. Biventricular pacemaker upgrade was performed after that because his heart function did not improve with optimal medical therapy. His electrocardiography showed narrow QRS waves after he had this procedure (Figure 3), and his chest X-ray confirmed that his cardiac device leads were in a proper position (Figure 4).

\section{DISCUSSION}

Right ventricular pacing altered the function and the geometry of the left ventricle function. This resulted primarily from the left ventricular dyssynchrony induced by functional left bundle branch block, especially among patients with predominant pacing [8-10]. As demonstrated in animal studies, right ventricular pacing reduces tissue perfusion and increases norepinephrine release, which leads to myofibrillar cellular disarray and dystrophic calcifications [4, 5]. Therefore, those patients also have a higher incidence of atrial fibrillation, ventricular arrhythmia, heart failure hospitalization, and death $[8,11]$. Nevertheless, this disease does not occur in all patients with right ventricular pacing despite the fact that left ventricular dyssynchrony is very common among those patients [12]. It is unclear whether patients with pacing-induced cardiomyopathy (PICM) have certain factors that increase their risk for this disease.

To the best of our knowledge, this is the first case report discussing an acute and severe form of PICM. In this case, it was unclear why this patient manifested with a serious

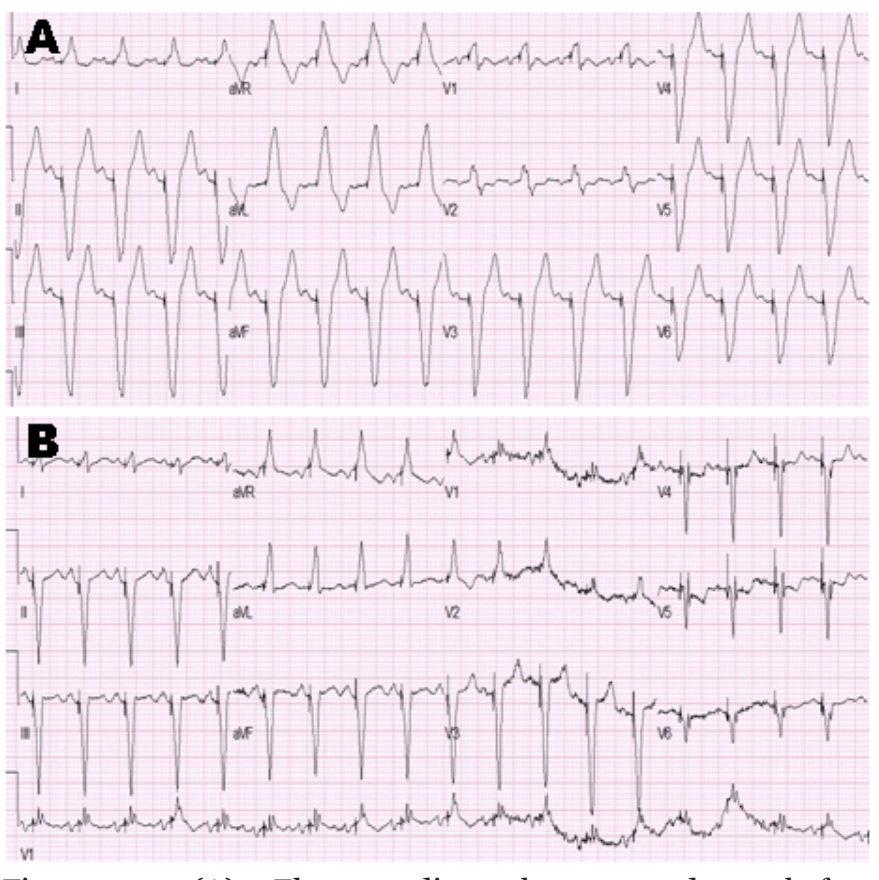

Figure 3: (A) Electrocardiography was done before biventricular cardiac device upgrade showing wide QRS, (B) Electrocardiography was done after biventricular device upgrade showings narrow QRS due to biventricular pacing. 

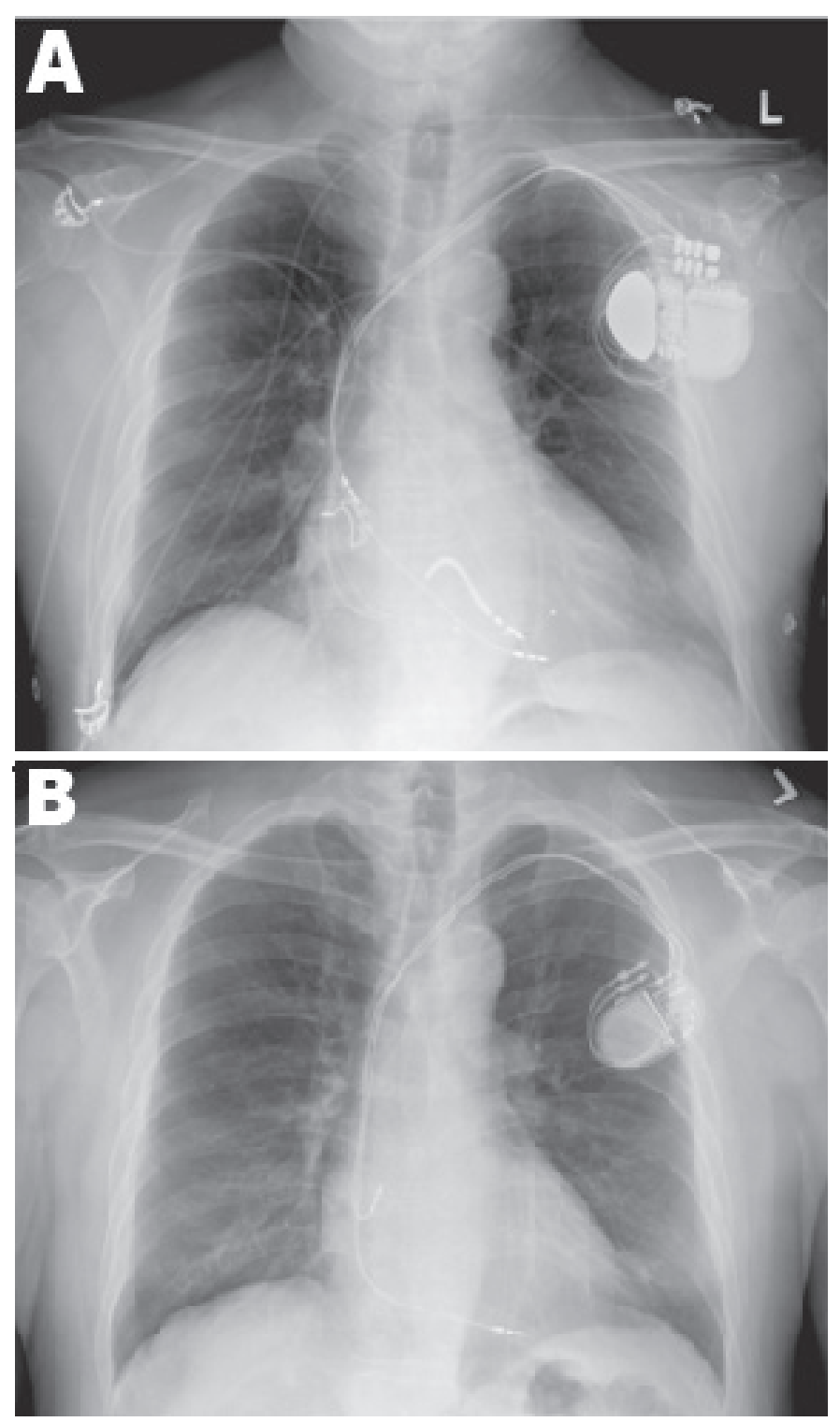

Figure 4: (A) X-rays done after pacemaker device placement, and (B) X-ray done after biventricular device upgrade.

and intense form of this disease or whether biventricular pacing could have prevented this disease in this patient. Several studies have shown that, in comparison with right ventricular pacing, biventricular pacing preserves and improves left ventricular function in patients with normal (>40\%) and low (<40\%) ejection fractions [13, 14]. Yu et al. found no significant differences in the rates of hospitalization or death between right ventricular pacing and biventricular pacing in patients that have a normal ejection fraction (45\%) and an indication for pacing [7]. However, Curtis et al. found significant difference in the rate of hospitalization but no difference in the rate of death in patients who have indications for pacing with atrioventricular block, class I, II, or III heart failure, and ejection fraction less than 50\% [15]. We think, that this patient had a serious and intense form of this disease because he had subendocardial inflammation and fibrosis before pacemaker placement, which might increase his risk to develop PICM. He also had risk factors for PICM including being male and having a low ejection fraction and wide QRS. The rule of cardiac MRI scan in predicting PICM among patients with normal ejection fraction (45\%) and atrioventricular block remains unclear to determine whether certain groups of patients might benefit from biventricular pacing instead of a right ventricular pacemaker.

Patients with PICM are usually treated effectively with medical therapy as well as cardiac resynchronization therapy (CRT). Schwerg et al. showed in a recent clinical study that CRT significantly improves the function of left ventricular function among patients with PICM who received an optimal medical therapy. In this study, patients who declined a CRT upgrade did not show any significant improvement in their left ventricular function within one year of optimal medical therapy [16]. Our patient's device was upgraded to CRT because he did not improve with an optimal medical therapy. An echocardiogram will be repeated within three months to evaluate the effect of CRT on his left ventricular function.

\section{CONCLUSION}

Pacing-induced cardiomyopathy is defined as a $10 \%$ reduction in the left ventricular ejection fraction over one year after pacemaker implantation, resulting in an ejection fraction of less than 50\%. Biventricular pacing preserves and improves the left ventricular function in patients with normal $(>40 \%)$ and low (<40\%) ejection fractions. Further studies are warranted to confirm the prognostic effect of cardiac MRI scan in predicting pacinginduced cardiomyopathy in patients who have a normal ejection fraction (45\%) and an indication for pacing such as patients with atrioventricular block. Such groups of patients might benefit from biventricular pacing instead of a right ventricular pacemaker.

$$
* * * * * * * * *
$$

\section{Author Contributions}

Talal Alzahrani - Substantial contributions to conception and design, Acquisition of data, Analysis and interpretation of data, Drafting the article, Revising it critically for important intellectual content, Final approval of the version to be published

Sripooja Satya - Analysis and interpretation of data, Revising it critically for important intellectual content, Final approval of the version to be published

Marco Mercader - Analysis and interpretation of data, Revising it critically for important intellectual content, Final approval of the version to be published

\section{Guarantor}

The corresponding author is the guarantor of submission. 


\section{Conflict of Interest}

Authors declare no conflict of interest.

\section{Copyright}

(C) 2016 Talal Alzahrani et al. This article is distributed under the terms of Creative Commons Attribution License which permits unrestricted use, distribution and reproduction in any medium provided the original author(s) and original publisher are properly credited. Please see the copyright policy on the journal website for more information.

\section{REFERENCES}

1. Nathan DA, Center S, Wu CY, Keller W. An implantable synchronous pacemaker for the long term correction of complete heart block. Am J Cardiol 1963 Mar;11:362-7.

2. Kurtz SM, Ochoa JA, Lau E, et al. Implantation trends and patient profiles for pacemakers and implantable cardioverter defibrillators in the United States: 1993-2006. Pacing Clin Electrophysiol 2010 Jun 1;33(6):705-11.

3. Thambo JB, Bordachar $\mathrm{P}$, Garrigue $\mathrm{S}$, et al. Detrimental ventricular remodeling in patients with congenital complete heart block and chronic right ventricular apical pacing. Circulation 2004 Dec 21;110(25):3766-72.

4. Lee MA, Dae MW, Langberg JJ, et al. Effects of longterm right ventricular apical pacing on left ventricular perfusion, innervation, function and histology. J Am Coll Cardiol 1994 Jul;24(1):225-32.

5. Karpawich PP, Justice CD, Cavitt DL, Chang CH. Developmental sequelae of fixed-rate ventricular pacing in the immature canine heart: An electrophysiologic, hemodynamic, andhistopathologic evaluation. Am Heart J 1990 May;119(5):1077-83.

6. Khurshid S, Epstein AE, Verdino RJ, et al. Incidence and predictors of right ventricular pacing-induced cardiomyopathy. Heart Rhythm 2014 Sep;11(9):161925.

7. Yu CM, Chan JY, Zhang Q, et al. Biventricular pacing in patients with bradycardia and normal ejection fraction. N Engl J Med 2009 Nov 26;361(22):212334 .

8. Steinberg JS, Fischer A, Wang P, et al. The clinical implications of cumulative right ventricular pacing in the multicenter automatic defibrillator trial II. J Cardiovasc Electrophysiol 2005 Apr;16(4):359-65.

9. Dreger H, Maethner K, Bondke H, Baumann G, Melzer C. Pacing-induced cardiomyopathy in patients with right ventricular stimulation for $>15$ years. Europace 2012 Feb;14(2):238-42.

10. Wilkoff BL, Cook JR, Epstein AE, et al. Dual-chamber pacing or ventricular backup pacing in patients with an implantable defibrillator: The Dual Chamber and VVI Implantable Defibrillator (DAVID) Trial. JAMA 2002 Dec 25;288(24):3115-23.

11. Sweeney MO, Hellkamp AS, Ellenbogen KA, et al. Adverse effect of ventricular pacing on heart failure and atrial fibrillation among patients with normal baseline QRS duration in a clinical trial of pacemaker therapy for sinus node dysfunction. Circulation 2003 Jun 17;107(23):2932-7.

12. Chen L, Hodge D, Jahangir A, et al. Preserved left ventricular ejection fraction following atrioventricular junction ablation and pacing for atrial fibrillation. $\mathrm{J}$ Cardiovasc Electrophysiol 2008 Jan;19(1):19-27.

13. Lieberman R, Padeletti L, Schreuder J, et al. Ventricular pacing lead location alters systemic hemodynamics and left ventricular function in patients with and without reduced ejection fraction. J Am Coll Cardiol 2006 Oct 17;48(8):1634-41.

14. Kindermann M, Hennen B, Jung J, Geisel J, Böhm M, Fröhlig G. Biventricular versus conventional right ventricular stimulation for patients with standard pacing indication and left ventricular dysfunction: the Homburg Biventricular Pacing Evaluation (HOBIPACE). J Am Coll Cardiol 2006 May 16;47(10):1927-37.

15. Curtis AB, Worley SJ, Adamson PB, et al. Biventricular pacing for atrioventricular block and systolic dysfunction. N Engl J Med 2013 Apr 25;368(17):158593.

16. Schwerg M, Dreger H, Poller WC, Dust B, Melzer C. Efficacy of optimal medical therapy and cardiac resynchronization therapy upgrade in patients with pacemaker-induced cardiomyopathy. J Interv Card Electrophysiol 2015 Dec;44(3):289-96.
Access full text article on other devices

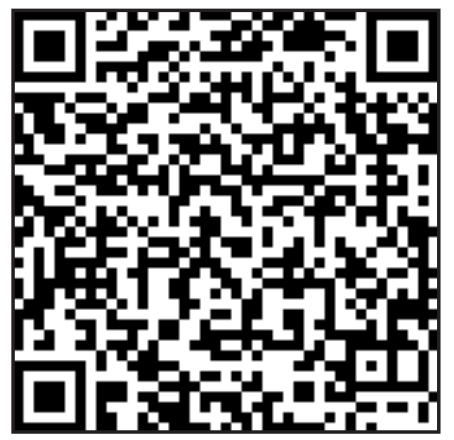

Access PDF of article on other devices

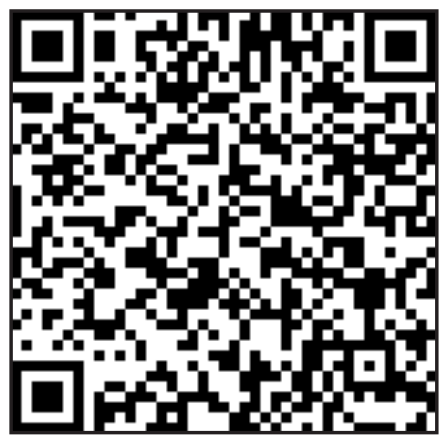

